New windows on massive stars: asteroseismology, interferometry, and spectropolarimetry

Proceedings IAU Symposium No. 30\%, 2014

G. Meynet, C. Georgy, J. H. Groh \& Ph. Stee, eds.

(C) International Astronomical Union 2015 doi:10.1017/S1743921314007236

\title{
Magnetic CP stars in Orion OB1 association
}

\section{Iosif I. Romanyuk and Eugene A. Semenko}

Special Astrophysical Observatory of the Russian Academy of Sciences, Nizhnii Arkhyz,

Russian Federation, 369167

email: roman@sao.ru, sea@sao.ru

\begin{abstract}
We present the overview of an observational program carring out on the 6-m telescope of Special Astrophysical Observatory from 2012. This program aims the searches of new Bp stars with surface magnetic field and the detailed study of known magnetic CP star from the stellar association Ori OB1. HD 34736 is the most interesting star that was found as magnetic within the program recently.
\end{abstract}

Keywords. stars: evolution, open clusters and associations: general, stars: magnetic fields, stars: chemically peculiar

\section{General results}

A total of $85 \mathrm{CP}$ stars of various types are identified among 814 members of the Orion OB1 association. The fraction of CP stars decreases with age for different subgroups from $15.1 \%$ in the youngest subgroup (b) to $7.7 \%$ in the oldest one (a).

We selected 59 Bp stars, which account for $13.4 \%$ of the total number of B type stars in the association. The fraction of peculiar B-type stars in the Orion OB1 association
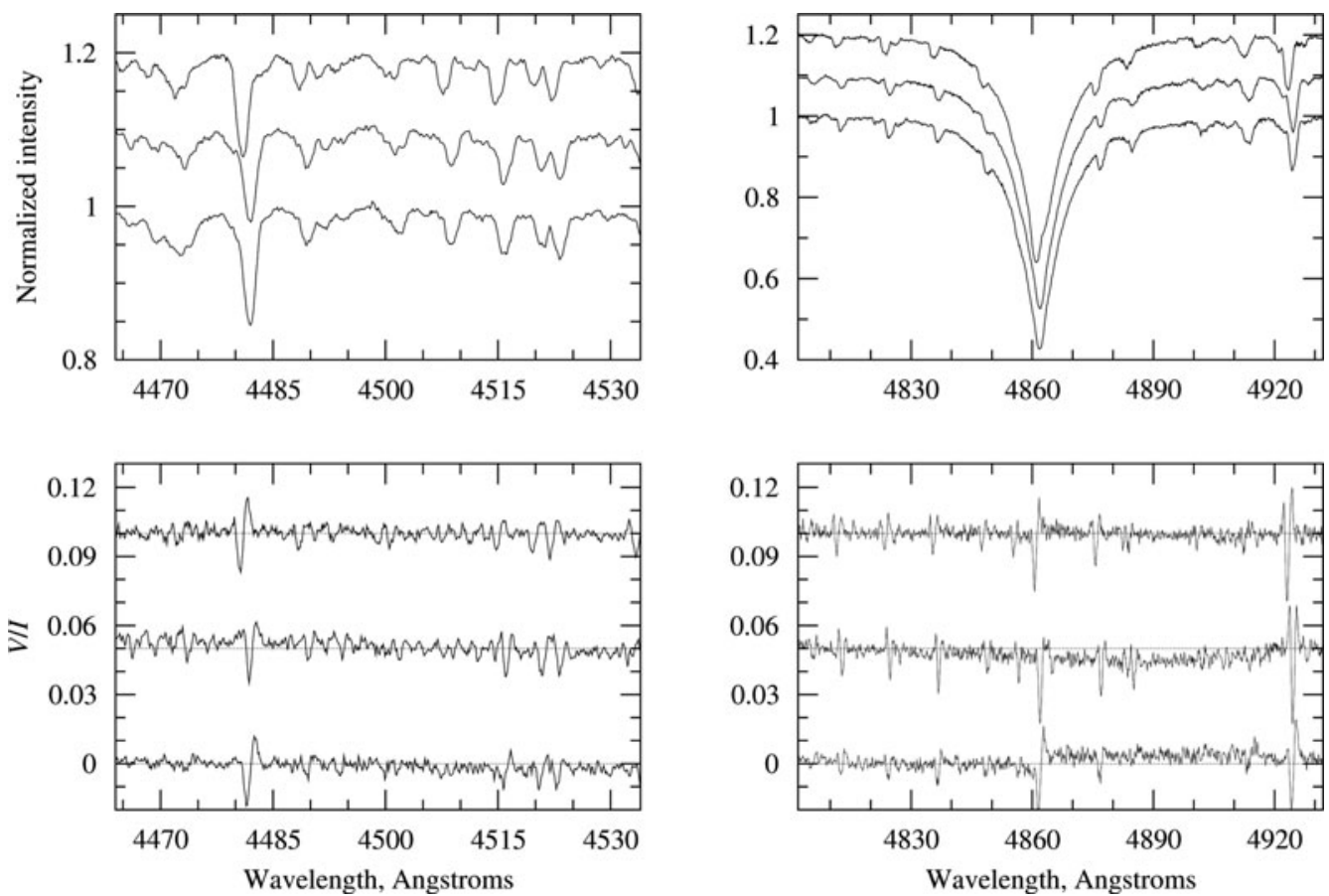

Figure 1. Changes in intensity spectrum (top panels) and $V$ Stockes parameter (bottom) during the observations in October and December, 2013. 

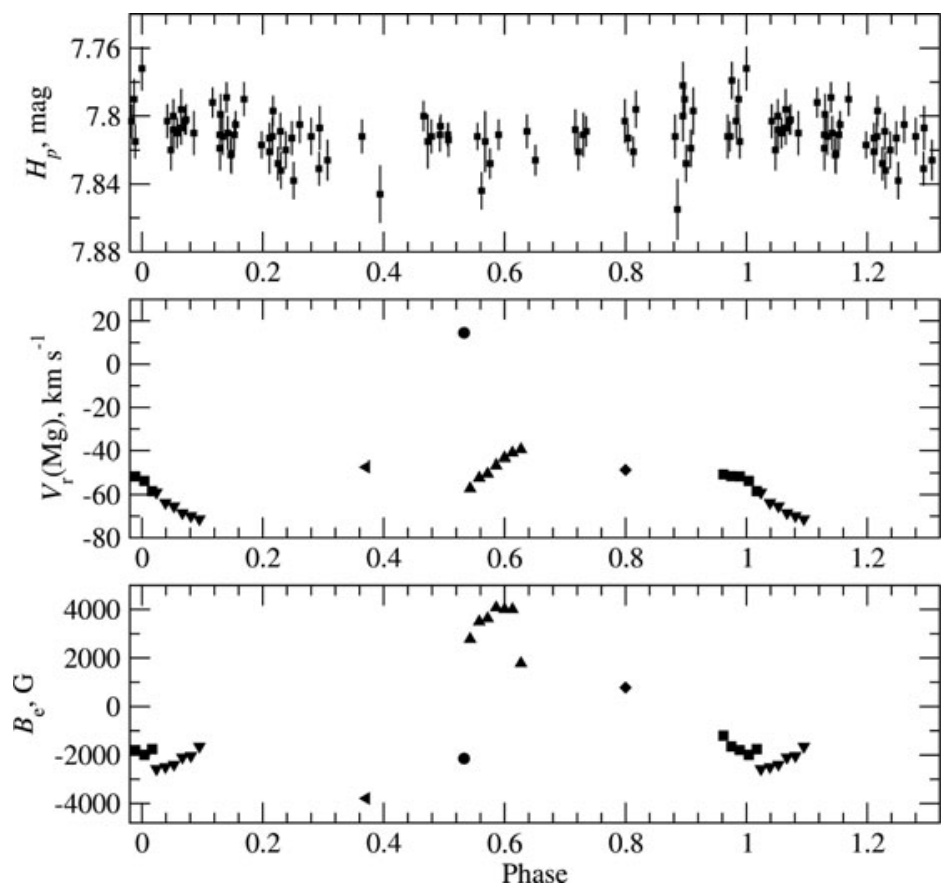

Figure 2. Individual measurements of (from top to bottom) HIPPARCOS photometry, radial velocity as measured from MgII 4481 1.0808 days. Individual observational sets are marked by different symbols. Errors in $V_{r}$ and $B_{e}$ measurement are comparable with corresponding markers size.

is found to be twice higher than of peculiar A-type stars. Magnetic fields are found in 23 stars, 17 of them are objects with anomalous helium lines. Magnetic stars show a well-defined tendency to concentrate in the central region of association. No significant differences are found between the field strength in the Bp type stars of the association and Bp type field stars (Romanyuk et al. 2013).

\section{HD 34736}

Spectropolarimetric observations were carried out with the $6 \mathrm{~m}$ Russian telescope. We acquired Zeeman spectra for all $59 \mathrm{Bp}$ stars from Orion OB1 association. Strong magnetic field was discovered on a star HD 34736 (Fig. 1). Its longitudinal field $B_{e}$ exceeds -4500 G (Semenko et al. 2014). Hydrogen line profile corresponds to the effective temperature of about $13700 \mathrm{~K}$. The variable radial velocity of some spectral lines and the presence of lines signatures of at least one additional component testify that HD 34736 is a short periodic binary system. We found the best orbital period has to be about 1.08 days (Fig.2).

Period of longitudinal magnetic field $B_{e}$ changes is not in coincidence with orbital one. Measured projected rotational velocity of magnetic component is $73 \mathrm{~km} \mathrm{~s}^{-1}$.

\section{References}

Romanyuk, I. I., Semenko, E. A., Yakunin, I. A., \& Kudryavtsev, D. O. 2013, Astrophysical Bulletin 68, 300

Semenko, E. A., Romanyuk, I. I., Kudryavtsev, D. O., \& Yakunin, I. A. 2014, Astrophysical Bulletin 69, 191 